\title{
A Totient function inequality
}

\author{
N. A. Carella \\ York College-CUNY, New York City, NY 11451, USA \\ *Corresponding author E-mail: pobox5050@gmail.com
}

\begin{abstract}
A new unconditional inequality of the totient function is contributed to the literature. This result is associated with various unsolved problems about the distribution of prime numbers.

Keywords: Totient function, Prime numbers, Nicolas inequality, highly composite numbers, and Riemann hypothesis.
\end{abstract}

\section{Introduction}

The totient function $\phi(N)=\#\{n<N: \operatorname{gcd}(n, N)=1\}$, which counts the number of relatively prime integers less than $N$, is a sine qua non in number theory. It and its various generalizations appear everywhere in the mathematical literature. The product form representation

$$
\phi(N)=N \prod_{p \mid N}(1-1 / p)
$$

unearths its intrinsic link to the distribution of the prime numbers. The totient function $\varphi(N)$ is an oscillatory function, its value oscillates from its maximum $\varphi(N)=N-1$ at prime integers $N$ to its minimum $\varphi(N)=N / c_{0} \log \log N$, at the primorial integers $N=2^{v_{1}} \cdot 3^{v_{2}} \cdots p_{k}^{v_{k}}$, where $p_{i}$ is the $k$ th prime, $v_{i} \geq 1$, and $c_{0}>1$ is a constant. The new contributions to the literature are the unconditional estimates stated below.

Theorem 1: Let $p_{i}$ be the kth prime, and let $N_{k}=2 \cdot 3 \cdot 5 \cdot p_{k}, k \geq 1$. Then $N_{k} / \phi\left(N_{k}\right)>e^{\gamma} \log \log N_{k}$ for all sufficiently large primorial integer $N_{k}$.

This unconditional result is consistent with the Riemann hypothesis, and seems to prove the Nicolas inequality, Theorem 4 below, for all sufficiently large integers. Just a finite number of cases of primorial integers $N_{k} \leq N_{0}$ remain unresolved as possible counterexamples of the inequality.

Theorem 2: The function $\phi(N) / N>c_{0} / \log \log \log N$ for almost every integer $N \geq 1$, and $c_{0}>0$ constant.

Currently the best unconditional estimate of this arithmetical function in the literature is the followings:

Theorem 3: ([13]) Let $N \in \mathbb{N}$, then $N / \phi(N)<e^{\gamma} N \log \log N+5 /(2 \log \log N)$ with one exception for $N=2 \cdot 323$. On the other hand, there are several conditional criteria; one of these is listed below.

Theorem 4: ([12]) Let $N_{k}=2 \cdot 3 \cdot 5 \cdot p_{k}$ be the product of the first $k$ primes.

1) If the Riemann Hypothesis is true then $N_{k} / \phi\left(N_{k}\right)>e^{\gamma} \log \log N_{k}$ for all $k \geq 1$.

2) If the Riemann Hypothesis is false then both $N_{k} / \phi\left(N_{k}\right)<e^{\gamma} \log \log N_{k}$ and $N_{k} / \phi\left(N_{k}\right)>e^{\gamma} \log \log N_{k}$ occur for infinitely many $k \geq 1$.

Some related and earlier works on this topic include the works of Ramanujan, Erdos, and other on abundant numbers, see [11], [2], and recent related works appeared in [2], [3], [9], [14], and [20]. 
The next section covers some background materials focusing on some finite sums over the prime numbers and some associated and products. The proofs of Theorems 1 and 2 are given in the last two sections respectively.

\section{Background materials}

This section provides a survey of supporting materials. An effort was made to have a self-contained paper as much as possible, but lengthy proofs available in the literature are omitted.

\subsection{Sums and products over the primes.}

The most basic finite sum over the prime numbers is the prime harmonic sum $\sum_{n \leq x} p^{-1}$. The refined estimate of this finite sum, stated below, is a synthesis of various results due to various authors. The earliest version $\sum_{n \leq x} p^{-1}=\log \log x+B_{1}+O(1 / \log x)$ is due to Mertens, see [17].

Theorem 5: Let $x \geq 2$ be a sufficiently large number. Then

$\sum_{p \leq x} \frac{1}{p}=\left\{\begin{array}{lc}\log \log x+B_{1}+O\left(e^{-c(\log \log x)^{1 / 2}}\right), & \text { unconditionally, } \\ \log \log x+B_{1}+O\left((\log x)^{-1 / 2}\right), & \text { conditional on the Riemann hypothesis, } \\ \log \log x+B_{1}+\Omega_{ \pm}\left((\log x)^{-1 / 2} \log \log \log x / \log x\right), & \text { unconditional oscillations, }\end{array}\right.$

Where B1 $=.2614972128$

Proof: Use the integral representation of the finite sum

$$
\sum_{p \leq x} \frac{1}{p}=\int_{c}^{x} \frac{d \pi(t)}{t}
$$

Where $c>1$ is a small constant. Moreover, the prime counting function $(x)=\#\{p \quad x: p$ is prime $\}$ has the form

$$
\pi(x)= \begin{cases}l i(x)+O\left(x e^{-c(\log x)^{1 / 2}}\right), & \text { unconditionally, } \\ l i(x)+O\left(x^{1 / 2} \log x\right), & \text { conditional on the Riemann hypothesis, } \\ l i(x)+\Omega_{ \pm}\left(x^{1 / 2} \log \log \log x / \log x\right), & \text { unconditional oscillations. }\end{cases}
$$

The unconditional part of the prime counting formula arises from the delaVallee Poussin form of the prime number theorem $\pi(x)=l i(x)+O\left(x e^{-c(\log x)^{1 / 2}}\right)$, see [10, p. 179], the conditional part arises from the Riemann form of the prime number theorem $\pi(x)=l i(x)+O\left(x^{1 / 2} \log x\right)$, and the unconditional oscillations part arises from the Littlewood form of the prime number theorem $\pi(x)=l i(x)+\Omega_{ \pm}\left(x^{1 / 2} \log \log \log x / \log x\right)$, consult [7, p. 51], [10, p. 479] et cetera. Now replace the logarithm integral $l i(x)=\int_{0}^{x}(t \log t)^{-1} d t$, and the appropriate prime counting measure $d \pi(t)$, and simplify the integral.

The proof of the unconditional part of this result is widely available in the literature; see [6], [10], [16], et cetera. The omega notation $f(x)=g(x)+\Omega_{ \pm}(h(x))$ means that both $f(x)>g(x)+c_{0} h(x)$ and $f(x)<g(x)-c_{0} h(x)$ occur infinitely often as $x \rightarrow \infty$, where $c_{0}>0$ is a constant, see [10, p. 5], [18].

As an application of the last result, there is the following interesting product:

Theorem 6: Let $x \in \mathbb{R}$ be a large real number, then

$\prod_{p \leq x}\left(1-\frac{1}{p}\right)^{-1}=\left\{\begin{array}{l}e^{\gamma} \log x+O\left(e^{-c(\log x)^{1 / 2}}\right), \\ e^{\gamma} \log x+O\left(x^{-1 / 2}\right), \\ e^{\gamma} \log x+\Omega_{ \pm}\left(x^{-1 / 2} \log \log \log x / \log x\right),\end{array}\right.$

unconditionally, conditional on the Riemann hypothesis, unconditional oscillations,

Proof: Consider the logarithm of the product 


$$
\begin{aligned}
\log \prod_{p \leq x}(1-1 / p)^{-1} & =\sum_{p \leq x} \frac{1}{p}+\sum_{p \leq x} \sum_{n \geq 2} \frac{1}{n p^{n}} \\
& =\sum_{p \leq x} \frac{1}{p}+\gamma-B_{1}+O\left(\frac{1}{x}\right),
\end{aligned}
$$

where the Euler constant is defined by $\gamma=\lim _{x \rightarrow \infty} \sum_{n \leq x}\left(n^{-1}-\log n\right)=0.577215665 \ldots$, and the Mertens constant is defined by $B_{1}=\gamma+\sum_{p \geq 2}(\log (1-1 / p)+1 / p)=.2614972128 \ldots$, see [6, p. 466]. The last equality in (6) stems from the power series expansion $B_{1}=\gamma-\sum_{p \geq 2} \sum_{n \geq 2}\left(n p^{n}\right)^{-1}$, which yields

$$
\begin{aligned}
\sum_{p \leq x} \sum_{n \geq 2} \frac{1}{n p^{n}} & =\gamma-B_{1}-\sum_{p>x} \sum_{n \geq 2} \frac{1}{n p^{n}} \\
& =\gamma-B_{1}+O\left(\frac{1}{x}\right)
\end{aligned}
$$

Applying Theorem 5 returns

$$
\log \prod_{p \leq x}\left(1-\frac{1}{p}\right)^{-1}= \begin{cases}\gamma+\log \log x+O\left(e^{-c(\log x)^{1 / 2}}\right), & \text { unconditionally, } \\ \gamma+\log \log x+O\left(x^{-1 / 2}\right), & \text { conditional on the Riemann hypothesis, } \\ \gamma+\log \log x+\Omega_{ \pm}\left(x^{-1 / 2} \log \log \log x / \log x\right), & \text { unconditional oscillations. }\end{cases}
$$

And reversing the logarithm completes the verification.

The third part in (5) above simplifies the proof given in [5] of the following result:

The quantity

$$
\left(x^{1 / 2} \log x\right)\left(\prod_{p \leq x}(1-1 / p)^{-1}-e^{\gamma} \log x\right)
$$

attains arbitrary large positive and negative values as $x \rightarrow \infty$.

\section{An estimate of the Totient function}

The proof of Theorem 3 on the extreme values of the arithmetic function $N / \phi(N)$ relies on the oscillation theorem of the finite prime product $\prod_{p \leq x}(1-1 / p)^{-1}$. This technique leads to a concise and simpler proof. A completely elementary proof, but longer, and not based on the oscillation theorem was presented in the earlier version of this paper.

Theorem 1: Let $N \in \mathbb{N}$ be a primorial integer, then $N / \varphi(N)>6 \pi^{-2} e^{\gamma} \log \log N$ holds unconditionally for all sufficiently large $N=2 \cdot 3 \cdot 5 \cdot p_{k}$.

Proof: Theorem 6 implies that the product

$$
\prod_{p \leq x}(1-1 / p)^{-1}=e^{\gamma} \log x+\Omega_{ \pm}\left(\frac{\log \log \log x}{x^{1 / 2} \log x}\right) \text {. }
$$

In particular, it follows that

$\prod_{p \leq x}(1-1 / p)^{-1}>e^{\gamma} \log x+c_{0} \frac{\log \log \log x}{x^{1 / 2} \log x}$

And

$\prod_{p \leq x}(1-1 / p)^{-1}<e^{\gamma} \log x-c_{0} \frac{\log \log \log x}{x^{1 / 2} \log x}$ 
Occur infinitely often as $x \rightarrow \infty$, where $c_{0}>0, c_{1}>0$, and $c_{2}>0$ are constants. It shows that $\prod_{p \leq x}(1-1 / p)^{-1}$ oscillates infinitely often, symmetrically about the line $e \log x$ as $x \rightarrow \infty$.

To rewrite the variable $x \geq 1$ in terms of the integer $N$, recall that the Chebychev function satisfies $\vartheta(x)=\sum_{p \leq x} \log p \leq c x, c_{1}>1$, see [15]. The properties of this function lead to

$\log N_{k}=\sum_{p \leq p_{k}} \log p=\vartheta\left(p_{k}\right)$, and $\vartheta\left(p_{k}\right)=p_{k}+o\left(p_{k}\right) \leq c_{1} \log N_{k}$.

So it readily follows that $p_{k} \leq x=c_{1} \log N_{k}$. Moreover, since the maxima of the sum of divisors function

$\frac{\sigma(N)}{N}=\prod_{p^{\alpha} \| N}\left(1+\frac{1}{p}+\frac{1}{p^{2}}+\cdots \frac{1}{p^{\alpha}}\right) \leq \prod_{p \mid N}(1-1 / p)^{-1}$,

where the symbol $p^{\alpha} \| N$ denotes the maximal prime power divisor of $N$, occur at the colossally abundant integers $N=2^{v_{1}} \cdot 3^{v_{2}} \cdots p_{k}^{v_{k}}$, and $v_{1} \geq v_{2} \geq \cdots \geq v_{k} \geq 1$, see [2], [3], [9], [11], it follows that the maxima of the inverse totient function $N / \varphi(N)$ occur at the squarefree primorial integers $N_{k}=2 \cdot 3 \cdot 5 \cdots p_{k}$. Therefore, expressions (10) and (11) implies that

$$
\begin{aligned}
\frac{N_{k}}{\varphi\left(N_{k}\right)} & =\prod_{p \leq c_{1} \log N_{k}}(1-1 / p)^{-1} \\
& >e^{\gamma} \log \log N_{k}+c_{2} \frac{\log \log \log \log N_{k}}{\left(\log N_{k}\right)^{1 / 2} \log \log N_{k}} \\
& >e^{\gamma} \log \log N_{k}
\end{aligned}
$$

as the primorial integer $N_{k}=2 \cdot 3 \cdot 5 \cdot p_{k}$ tends to infinity.

\section{Probabilistic properties}

The natural density function $B(t)=\lim _{N \rightarrow \infty} \#\{n \leq N: N / \phi(N) \geq t\} / N$

Is known to be a continuous function of $t \geq 0$. Some recent works have established the exact asymptotic expression

$B(t)=\exp \left(-e^{e^{-\gamma} t}\left(1+O\left(1 / t^{2}\right)\right)\right)$

As $t$ tends to infinity, see [19], [20].

The evaluation of the natural density (15) at $t=e^{\gamma} \log \log N$ as $N \rightarrow \infty$ suggests that the Nicolas inequality should be $e^{\gamma} \log \log N_{k}>N_{k} / \phi\left(N_{k}\right)>e^{\gamma+\alpha(k)} \log \log N_{k}^{1-\varepsilon}$. The numerical data are compiled in [8].

Now, note that the evaluation at $t=e^{\gamma} \log \log \log N$ as $N \rightarrow \infty$ yields the density function

$$
\begin{aligned}
B\left(t=e^{\gamma} \log \log \log N\right) & =\exp \left(-e^{e^{-\gamma} t}\left(1+O\left(1 / t^{2}\right)\right)\right) \\
& =\frac{1}{\log N}\left(1+O\left(1 /(\log \log \log N)^{2}\right)\right) .
\end{aligned}
$$

Consequently, the subset of integers $N \geq 1$ such that $N / \phi(N)>c_{0} \log \log \log N$ has zero density with respect to the set of integers $\mathbb{N}$. A simple proof of this result is included here.

Theorem 2: The function $\phi(N)>c_{0} N / \log \log \log N$ for almost every integer $N \geq 1$, and $c_{0}>0$ constant.

Proof: The prime divisors counting function satisfies $\omega(N)=\#\{p \mid N\} \leq c_{1} \log \log N$ for almost every integer $N \geq 1$, this is Ramanujan Theorem. In addition,

$$
\prod_{p \mid N}(1-1 / p) \geq \prod_{p \leq x}(1-1 / p) \geq \frac{e^{-\gamma}}{\log x}\left(1-\frac{1}{2 \log ^{2} x}\right),
$$


where $x \geq 2$ is a suitable real number, holds for every integer $N \geq 1$, this is Mertens Theorem. Furthermore, by the Prime Number Theorem, the $n$th prime $p_{n} \leq c_{2} n \log n$. In light of these facts, put

$x=c_{2}\left(c_{1} \log \log N\right) \log \left(c_{1} \log \log N\right)$

$\leq c_{3} \log \log N \log \log \log N$,

where $c_{1}, c_{2}, c_{3}, c_{4}$, are constants. Substituting (19) into the previous relation (18) implies that

$$
\begin{aligned}
\prod_{p \mid N}(1-1 / p) & \geq \prod_{p \leq p_{n}}(1-1 / p) \\
& \geq \frac{c_{4}}{\log \log \log N}
\end{aligned}
$$

holds for almost every integer $N \geq 1$. Ergo, the ratio $\phi(N) / N=\prod_{p \mid N}(1-1 / p) \geq c_{4} / \log \log \log N$ holds for almost every integer $N \geq 1$ as claimed.

Corollary 7: The function $\sigma(N)<c_{5} N \log \log \log N$ for almost every integer $N \geq 1$, and $c_{5}>0$ constant.

Proof: The sigma-phi identity, on the first line below, coupled with Theorem 2 lead to

$$
\begin{aligned}
\frac{\sigma(N)}{N} & =\frac{N}{\phi(N)} \prod_{p^{\alpha} \| N}\left(1-1 / p^{\alpha+1}\right) \\
& <\frac{N}{\phi(N)} \\
& <c_{5} N \log \log \log N
\end{aligned}
$$

where $c_{5}$ is a constant, holds for almost every integer $N \geq 1$.

\section{References}

[1] American Institute of Mathematics, aimath.org.

[2] L. Alaoglu and P. Erdos, on highly composite and similar numbers, Trans. Amer: Math. Soc. 56 (1944), 448-469.

[3] Briggs, K. Abundant numbers and the Riemann hypothesis. Experiment. Math. 15 (2006), no. 2, 251-256.

[4] William D. Banks, Derrick N. Hart, Pieter Moree, C. Wesley Nevans, The Nicolas and Robin inequalities with sums of two squares, arXiv: 0710.2424

[5] Diamond, H. G.; Pintz, J. (2009). "Oscillation of Mertens' product formula". J. de Theorie des Nombres de Bordeaux 21: $523-533$.

[6] G. H. Hardy and E. M. Wright, an Introduction to the Theory of Numbers, 5th ed., Oxford University Press, Oxford, 1979.

[7] Ivić, Aleksandar the Riemann zeta-function. The theory of the Riemann zeta-function with applications. A Wiley-Interscience Publication. John Wiley \& Sons, Inc., New York, 1985. ISBN: 0-471-80634-X.

[8] Tadej Kotnik, Computational Investigation of Five Problems in Analytic Number Theory, Preprint, 2006 available at www.

[9] Jeffrey C. Lagarias, An Elementary Problem Equivalent to the Riemann Hypothesis, arXiv:math/0008177.

[10] Montgomery, Hugh L.; Vaughan, Robert C. Multiplicative number theory. I. Classical theory. Cambridge Studies in Advanced Mathematics, 97. Cambridge University Press, Cambridge, 2007.

[11] S. Ramanujan, Highly composite numbers, Proc. London Math. Soc. 14 (1915), 347407

[12] J.-L. Nicolas, Petites valeurs de la fonction d'Euler, J. Number Theory 17 (1983) 375-388.

[13] G. Robin, Grandes valeurs de la fonction somme des diviseurs et hypoth`ese de Riemann, J. Math. Pures Appl. (9) 63 (1984) $187-213$.

[14] Y.-J. Choie, N. Lichiardopol, P. Moree, P. Sole, On Robin's criterion for the Riemann Hypothesis, arXiv:math/0604314.

[15] Shapiro, Harold N. Introduction to the theory of numbers. Pure and Applied Mathematics. A Wiley-Interscience Publication. New York, 1983.

[16] G. Tenenbaum, Introduction to analytic and probabilistic number theory, Cambridge Studies in Advanced Mathematics 46, (Cambridge University Press, Cambridge, 1995.

[17] Mark B. Villarino, Mertens' Proof of Mertens' Theorem, arXiv:math/0504289v3.

[18] Wikipedia.org.

[19] Weingartner, Andreas. The distribution functions of \$lsigma (n)/n\$ and \$n/phi (n) \$. Proc. Amer. Math. Soc. 135 (2007), no. 9 , $2677-2681$.

[20] Weingartner, Andreas. The limiting distribution of the divisor function. J. Number Theory 129 (2009), no. 6, 1432-1442.

[21] Marek Wójtowicz, Robin's inequality and the Riemann hypothesis, Proc. Japan Acad. Ser. A Math. Sci. Volume 83, Number 4 (2007), 47-49. 